\title{
APPROXIMATE SIMULATION RELATIONS FOR HYBRID SYSTEMS ${ }^{1}$
}

\author{
Antoine Girard* A. Agung Julius* \\ George J. Pappas* \\ * Department of Electrical and Systems Engineering \\ University of Pennsylvania \\ Philadelphia, PA 19104 \\ \{agirard,agung,pappasg\}@seas.upenn.edu
}

\begin{abstract}
Approximate simulation relations have recently been introduced as a powerful tool for the approximation of discrete and continuous systems. In this paper, we extend this notion to hybrid systems. Using the so-called simulation functions, we develop a computationally effective characterization of approximate simulation relations which can be used for hybrid systems approximation. An example of application in the context of safety verification is shown.
\end{abstract}

Keywords: Approximation of hybrid systems, Approximate simulation relation.

\section{INTRODUCTION}

Approximation of purely discrete systems has traditionally been based on language inclusion and equivalence with notions such as simulation or bisimulation relations (Clarke et al., 2000). These concepts have been useful for simplifying problems such as safety verification or controller synthesis. More recently, they have been extended to the framework of continuous and hybrid systems (Pappas, 2003; Haghverdi et al., 2005) allowing the approximation of systems in a unified (discrete/continuous) manner.

When dealing with continuous and hybrid systems, typically observed over the real numbers with possibly noisy observations, the usual notions based on exact language inclusion is quite restrictive and not robust. The notion of language approximation is much more adequate in this context. In (Girard and Pappas, 2005c), we proposed a framework for system approximation

1 This research is partially supported by the Région Rhône-Alpes (Projet CalCel) and the NSF Presidential Early CAREER (PECASE) Grant 0132716. based on approximate versions of simulation relations. Instead of requiring that the observations of a system and its approximation are and remain equal, we require that they are and remain arbitrarily close. This approach not only defines more robust relations between systems but also allows more significant complexity reduction in the approximation process. In (Girard and Pappas, 2005a; Girard and Pappas, 2005b), this framework has been applied to constrained linear systems and nonlinear autonomous systems. Computational methods have been developed to quantify the distance between two systems. In (Julius et al., 2006; Julius, 2006), the theoretical and computational frameworks have been extended to handle stochastic dynamical and hybrid systems (with purely stochastic jumps). Related work on approximate versions of simulation and bisimulation relations has been done for quantitative transition systems (de Alfaro et al., 2004) or labeled Markov processes (Desharnais et al., 2004).

In this paper, we apply our approximation framework to hybrid systems. Using the so-called simulation functions (Girard and Pappas, 2005c), we 
develop a computationally effective characterization of approximate simulation relations which can be used for hybrid systems approximation. An example of application in the context of safety verification is shown.

\section{APPROXIMATION OF TRANSITION SYSTEMS}

In this section, we summarize the notion of approximate simulation relations for labeled transition systems as developed in (Girard and Pappas, 2005c). Labeled transition systems allow to model in a unified framework, discrete, continuous and hybrid systems. They can be seen as graphs, possibly with an infinite number of states or transitions.

Definition 2.1. A labeled transition system with observations is a tuple $T=\left(Q, \Sigma, \rightarrow, Q^{0}, \Pi,\langle\langle\rangle\rangle.\right)$ that consists of:

- a set $Q$ of states,

- a set $\Sigma$ of labels,

- a transition relation $\rightarrow \subseteq Q \times \Sigma \times Q$,

- a set $Q^{0} \subseteq Q$ of initial states,

- a set $\Pi$ of observations, and

- an observation map $\langle\langle\rangle\rangle:. Q \rightarrow \Pi$.

A state trajectory of $T$ is a sequence of transitions,

$$
q^{0} \stackrel{\sigma^{0}}{\rightarrow} q^{1} \stackrel{\sigma^{1}}{\rightarrow} q^{2} \stackrel{\sigma^{2}}{\rightarrow} \ldots, \text { where } \mathrm{q}^{0} \in \mathrm{Q}^{0} .
$$

For a given initial state and sequence of labels, there may exist several state trajectories of $T$. Thus, the systems we consider are possibly nondeterministic. The associated external trajectory

$$
\pi^{0} \stackrel{\sigma^{0}}{\rightarrow} \pi^{1} \stackrel{\sigma^{1}}{\rightarrow} \pi^{2} \stackrel{\sigma^{2}}{\rightarrow} \ldots, \text { where } \pi^{\mathrm{i}}=\left\langle\left\langle\mathrm{q}^{\mathrm{i}}\right\rangle\right\rangle
$$

describes the evolution of the observations under the dynamics of the labeled transition system. The set of external trajectories of the labeled transition system $T$ is called the language of $T$. The subset of $\Pi$ reachable by the external trajectories of $T$ is noted $\operatorname{Reach}(T)$. An important problem for transition systems is the safety verification problem which consists in checking whether the reachable set $\operatorname{Reach}(T)$ intersects a set of observations $\Pi_{U}$ associated with unsafe states.

Exact simulation relations between two labeled transition systems require that their observations are (and remain) identical (Clarke et al., 2000). Approximate simulation relations are less rigid since they only require that the observations of both systems are (and remain) arbitrarily close. Let $T_{1}=\left(Q_{1}, \Sigma_{1}, \rightarrow_{1}, Q_{1}^{0}, \Pi_{1},\langle\langle.\rangle\rangle_{1}\right)$ and $T_{2}=$ $\left(Q_{2}, \Sigma_{2}, \rightarrow_{2}, Q_{2}^{0}, \Pi_{2},\langle\langle\cdot\rangle\rangle_{2}\right)$ be two labeled transition systems with the same set of labels $\left(\Sigma_{1}=\right.$ $\left.\Sigma_{2}=\Sigma\right)$ and the same set of observations $\left(\Pi_{1}=\right.$
$\Pi_{2}=\Pi$ ). Let us assume that the set of observations $\Pi$ is a metric space; $d_{\Pi}$ denotes the metric on $\Pi$.

Definition 2.2. A relation $\mathcal{S}_{\delta} \subseteq Q_{1} \times Q_{2}$ is a $\delta$ approximate simulation relation of $T_{1}$ by $T_{2}$ if for all $\left(q_{1}, q_{2}\right) \in \mathcal{S}_{\delta}$ :

(1) $d_{\Pi}\left(\left\langle\left\langle q_{1}\right\rangle\right\rangle_{1},\left\langle\left\langle q_{2}\right\rangle\right\rangle_{2}\right) \leq \delta$,

(2) $\forall q_{1} \stackrel{\sigma}{\rightarrow} 1 q_{1}^{\prime}, \exists q_{2} \stackrel{\sigma}{\rightarrow} 2 q_{2}^{\prime}$ such that $\left(q_{1}^{\prime}, q_{2}^{\prime}\right) \in \mathcal{S}_{\delta}$.

Note that for $\delta=0$, we have the usual notion of exact simulation relation (Clarke et al., 2000).

Definition 2.3. $T_{2}$ approximately simulates $T_{1}$ with the precision $\delta$ (noted $T_{1} \preceq_{\delta} T_{2}$ ), if there exists $\mathcal{S}_{\delta}$, a $\delta$-approximate simulation relation of $T_{1}$ by $T_{2}$ such that for all $q_{1} \in \mathcal{Q}_{1}^{0}$, there exists $q_{2} \in \mathcal{Q}_{2}^{0}$ such that $\left(q_{1}, q_{2}\right) \in \mathcal{S}_{\delta}$.

If $T_{2}$ approximately simulates $T_{1}$ with the precision $\delta$ then the language of $T_{1}$ is approximated with precision $\delta$ by the language of $T_{2}$.

Theorem 2.4. (Girard and Pappas, 2005c) If $T_{1} \preceq_{\delta} T_{2}$, then for all external trajectories of $T_{1}$,

$$
\pi_{1}^{0} \stackrel{\sigma^{0}}{\rightarrow} \pi_{1}^{1} \stackrel{\sigma^{1}}{\rightarrow} \pi_{1}^{2} \stackrel{\sigma^{2}}{\rightarrow} \ldots,
$$

there exists an external trajectory of $T_{2}$ with the same sequence of labels

$$
\pi_{2}^{0} \stackrel{\sigma^{0}}{\rightarrow} \pi_{2}^{1} \stackrel{\sigma^{1}}{\rightarrow} \pi_{2}^{2} \stackrel{\sigma^{2}}{\rightarrow} \ldots
$$

such that for all $i \in \mathbb{N}, d_{\Pi}\left(\pi_{1}^{i}, \pi_{2}^{i}\right) \leq \delta$.

Approximation of transition systems based on approximate simulation relations is useful for solving the safety verification problem. Indeed, from Theorem 2.4, it is straightforward that if $T_{2}$ approximately simulates $T_{1}$ with the precision $\delta$ and $\operatorname{Reach}\left(T_{2}\right) \cap \mathcal{N}_{\Pi}\left(\Pi_{U}, \delta\right)=\emptyset\left(\right.$ where $\mathcal{N}_{\Pi}(., \delta)$ denotes the $\delta$-neighborhood for the metric $d_{\Pi}$ ), then $\operatorname{Reach}\left(T_{1}\right) \cap \Pi_{U}=\emptyset$. Therefore, the safety of $T_{1}$ can be verified using the approximate system $T_{2}$.

\section{HYBRID SYSTEMS AS TRANSITION SYSTEMS}

In this section, we show that hybrid systems can be formulated as transition systems. A hybrid system is defined as a tuple $H=(L, n, p, E, F, I n v, G$, $R, Q^{0}$ ) where

- $L$ is a finite set of locations or discrete states. $|L|$ denotes the number of elements of $L$. Without loss of generality, $L=\{1, \ldots,|L|\}$.

- $n: L \rightarrow \mathbb{N}$, where for every $l \in L, n_{l}$ is the dimension of the continuous state space in 
the location $l$. The set of states of the hybrid system is

$$
Q=\bigcup_{l \in L}\{l\} \times \mathbb{R}^{n_{l}} .
$$

- $p: L \rightarrow \mathbb{N}$, where for every $l \in L, p_{l}$ is the dimension of the continuous observation of the hybrid system in the location $l$. The set of observations of the hybrid system is

$$
\Pi=\bigcup_{l \in L}\{l\} \times \mathbb{R}^{p_{l}} .
$$

- $E \subseteq L \times L$ is the set of events or discrete transitions.

- $F=\left\{F_{l} \mid l \in L\right\}$ defines the continuous dynamics in each location. For each $l \in L$, $F_{l}$ is a triple $\left(f_{l}, g_{l}, U_{l}\right)$ where $f_{l}: \mathbb{R}^{n_{l}} \times$ $U_{l} \rightarrow \mathbb{R}^{n_{l}}, g_{l}: \mathbb{R}^{n_{l}} \rightarrow \mathbb{R}^{p_{l}}$ and $U_{l} \subseteq \mathbb{R}^{m_{l}}$ is a compact set of internal inputs accounting for disturbances and modelling uncertainties. While the discrete part of the state is $l$, the continuous part evolves according to

$$
\left\{\begin{array}{l}
\dot{x}(t)=f_{l}(x(t), u(t)), u(t) \in U_{l} \\
y(t)=g_{l}(x(t)) .
\end{array}\right.
$$

- Inv $=\left\{\operatorname{Inv}_{l} \mid l \in L\right\}$ defines an invariant set in each location. For each $l \in L, \operatorname{Inv}_{l} \subseteq \mathbb{R}^{n_{l}}$ constrains the value of the continuous part of the state while the discrete part is $l$.

- $G=\left\{G_{e} \mid e \in E\right\}$ defines the guard for each discrete transition. For each $e=\left(l, l^{\prime}\right) \in$ $E, G_{e} \subseteq I_{n v}$. The discrete transition $e$ is enabled when the continuous part of the state is in $G_{e}$.

- $R=\left\{R_{e} \mid e \in E\right\}$ defines the reset map for each discrete transition. For each $e=\left(l, l^{\prime}\right) \in$ $E, R_{e}: G_{e} \rightarrow 2^{I n v_{l^{\prime}}}$. When the event $e$ occurs, the continuous part of the state is reset using $R_{e}$.

- $Q^{0} \subseteq Q$ is the set of initial states:

$$
Q^{0}=\bigcup_{l \in L}\{l\} \times I_{l}^{0}, \text { with } I_{l}^{0} \subseteq \operatorname{Inv}_{l} .
$$

The semantics of a hybrid system is well established (see for instance (Alur et al., 2000)) and is not defined here. In the spirit of (Alur et al., 1995), we can derive from $H$ a nondeterministic transition system $T=\left(Q, \Sigma, \rightarrow, Q^{0}, \Pi,\langle\langle\rangle\rangle.\right)$ where the set of states $Q$, the set of observations $\Pi$, and the set initial states $Q^{0}$ are the same than in $H$. The set of labels is $\Sigma=\mathbb{R}^{+} \cup\{\tau\}$. The observation map is given by

$$
\langle\langle(l, x)\rangle\rangle=\left(l, g_{l}(x)\right) .
$$

The transition relation $\rightarrow$ is given by:

(1) continuous transitions:

For $t \in \mathbb{R}^{+},(l, x) \stackrel{t}{\rightarrow}\left(l, x^{\prime}\right)$ iff there exists a locally measurable function $u($.$) and an$ absolutely continuous function $z($.$) such that$ $z(0)=x, z(t)=x^{\prime}$ and for all $s \in[0, t]$,

$$
\dot{z}(s)=f_{l}(z(s), u(s))
$$

with $u(s) \in U_{l}$ and $z(s) \in \operatorname{Inv}_{l}$.

(2) discrete transitions:

$(l, x) \stackrel{\tau}{\rightarrow}\left(l^{\prime}, x^{\prime}\right)$ iff $\left(l, l^{\prime}\right)=e \in E, x \in G_{e}$ and $x^{\prime} \in R_{e}(x)$.

The set of observation $\Pi$ of a hybrid system is equipped with the following metric $d_{\Pi}$ :

$$
d_{\Pi}\left(\left(l_{1}, y_{1}\right),\left(l_{2}, y_{2}\right)\right)= \begin{cases}\left\|y_{1}-y_{2}\right\|, & \text { if } l_{1}=l_{2} \\ +\infty, & \text { if } l_{1} \neq l_{2}\end{cases}
$$

In the following, we show that our approximation framework based on approximate simulation relations can be applied to hybrid systems.

\section{APPROXIMATE SIMULATION RELATIONS FOR HYBRID SYSTEMS}

Let $H_{i}=\left(L_{i}, n_{i}, p_{i}, E_{i}, F_{i}, \operatorname{Inv}_{i}, G_{i}, R_{i}, Q_{i}^{0}\right),(i=$ $1,2)$ be two hybrid systems and $T_{i}=\left(Q_{i}, \Sigma_{i}, \rightarrow_{i}\right.$ , $\left.Q_{i}^{0}, \Pi_{i},\langle\langle.\rangle\rangle_{i}\right),(i=1,2)$ be the associated transition systems. We assume that $T_{1}$ and $T_{2}$ have the same set of observations $\Pi_{1}=\Pi_{2}=\Pi$. Particularly, this implies that the set of locations and the dimensions of the continuous observations are the same for both systems (i.e. $L_{1}=L_{2}=L$, $\left.p_{1}=p_{2}=p\right) .{ }^{2}$ We will further assume that the discrete dynamics of both systems are the same (i.e. $E_{1}=E_{2}=E$ ). The goal of the approximation process presented here is then essentially to simplify the continuous dynamics of the hybrid system $H_{1}$. In this section, we establish sufficient conditions so that $H_{2}$ approximately simulates $H_{1}$ and provide a method to evaluate the precision of the approximate simulation relation.

\subsection{Simulation functions}

Let $l \in L$, let $n_{1, l}, n_{2, l}$ be the dimensions of the continuous part of the state of $H_{1}$ and $H_{2}$ in the location $l$. Let $F_{1, l}=\left(f_{1, l}, g_{1, l}, U_{1, l}\right)$ and $F_{2, l}=\left(f_{2, l}, g_{2, l}, U_{2, l}\right)$ be the continuous dynamics of $H_{1}$ and $H_{2}$ associated to the location $l$. We define the following notations:

$$
\begin{gathered}
x=\left[\begin{array}{l}
x_{1} \\
x_{2}
\end{array}\right], f_{l}\left(x, u_{1}, u_{2}\right)=\left[\begin{array}{l}
f_{1, l}\left(x_{1}, u_{1}\right) \\
f_{2, l}\left(x_{2}, u_{2}\right)
\end{array}\right], \\
g_{l}(x)=g_{1, l}\left(x_{1}\right)-g_{2, l}\left(x_{2}\right) .
\end{gathered}
$$

In (Girard and Pappas, 2005c), we showed that approximate simulation relations could be characterized efficiently using the notion of simulation

\footnotetext{
2 The approximation of a hybrid systems by another hybrid systems with a smaller number of locations has been considered for systems with purely stochastic jumps (Julius, 2006). We will also consider this type of approximation for hybrid systems with non stochastic jumps in the future.
} 
function. In our context, this can be instantiated as follows.

Definition 4.1. $V_{l}: \mathbb{R}^{n_{1, l}} \times \mathbb{R}^{n_{2, l}} \rightarrow \mathbb{R}^{+}$is a simulation function of $F_{1, l}$ by $F_{2, l}$ if for all $x \in$ $\mathbb{R}^{n_{1, l}} \times \mathbb{R}^{n_{2, l},}$

$$
\begin{gathered}
V_{l}(x) \geq g_{l}(x)^{T} g_{l}(x), \\
\max _{u_{1} \in U_{1, l}} \min _{u_{2} \in U_{2, l}} \nabla V_{l}(x)^{T} f_{l}\left(x, u_{1}, u_{2}\right) \leq 0 .
\end{gathered}
$$

Remark 4.2. The concept of simulation function is related to robust control Lyapunov functions (Freeman and Kokotovic, 1996; Liberzon et al., 2002), though they slightly differ in spirit. Indeed, considering the input $u_{1}$ as a disturbance and the input $u_{2}$ as a control variable, the interpretation of equation (2) is that for all disturbances there exists a control such that the simulation function decreases during the evolution of the system. In this context, $u_{2}$ may have full knowledge (and be a function) of $u_{1}$. In comparison, a robust control Lyapunov function would require that there exists a control $u_{2}$ such that for all possible (and unknown) disturbances $u_{1}$ the function decreases during the evolution of the system. Therefore, robust control Lyapunov functions require stronger conditions than simulation functions.

Methods for the computation of simulation functions have been proposed for the class of constrained linear systems (Girard and Pappas, 2005a) and autonomous nonlinear systems (Girard and Pappas, 2005b). These methods are based on linear matrix inequalities, sum of squares programs and static games and are thus computationally effective. The computation of simulation functions for constrained linear systems has been implemented in the Matlab toolbox MATISSE ${ }^{3}$.

Simulation functions satisfy the following property which will be useful in characterizing approximate simulation relations for hybrid systems.

Proposition 4.3. For all $\left(x_{1}, x_{2}\right) \in \mathbb{R}^{n_{1, l}} \times \mathbb{R}^{n_{2, l}}$, for all inputs $u_{1}($.$) , there exists an input u_{2}$ (.) such that

$$
\forall t \in \mathbb{R}^{+}, V_{l}\left(z_{1}(t), z_{2}(t)\right) \leq V_{l}\left(x_{1}, x_{2}\right)
$$

where

$$
\dot{z}_{i}(t)=f_{i, l}\left(z_{i}(t), u_{i}(t)\right), z_{i}(0)=x_{i}, i=1,2 .
$$

Proof: Let us remark that

$$
\dot{V}_{l}(z(t))=\nabla V_{l}(z(t))^{T} f_{l}\left(z(t), u_{1}(t), u_{2}(t)\right)
$$

3 MATISSE: Metrics for Approximate TransItion Systems Simulation and Equivalence, Available from http://www. seas. upenn.edu/〜agirard/Software/MATISSE where $z(t)=\left[\begin{array}{lll}z_{1}(t) & z_{2}(t)\end{array}\right]^{T}$. Then, from equation $(2)$, it is clear that for all inputs $u_{1}($.$) , there exists$ an input $u_{2}($.$) such that \dot{V}_{l}(z(t)) \leq 0$.

\subsection{Approximate simulation relations}

In this section, we give a characterization of approximate simulation relations for hybrid systems. Let us assume that for each location $l \in L$, there exists a simulation function $V_{l}$ of the continuous dynamics $F_{1, l}$ by $F_{2, l}$. We define the following sets, for all $x_{1} \in \mathbb{R}^{n_{1, l}}, \beta \geq 0$,

$$
\mathcal{N}_{l}\left(x_{1}, \beta\right)=\left\{x_{2} \in \mathbb{R}^{n_{2, l}} \mid V_{l}\left(x_{1}, x_{2}\right) \leq \beta\right\} .
$$

Theorem 4.4. Let $\beta_{1}, \ldots, \beta_{|L|}$ be positive numbers such that

(a) for all $l \in L, \mathcal{N}_{l}\left(\operatorname{Inv}_{1, l}, \beta_{l}\right) \subseteq \operatorname{Inv}_{2, l}$,

(b) for all $e=\left(l, l^{\prime}\right) \in E, \mathcal{N}_{l}\left(G_{1, e}, \beta_{l}\right) \subseteq G_{2, e}$,

(c) for all $l \in L$,

$$
\beta_{l} \geq \max _{x_{1} \in I_{1, l}^{0}} \min _{x_{2} \in I_{2, l}^{0}} V_{l}\left(x_{1}, x_{2}\right),
$$

(d) for all $e=\left(l, l^{\prime}\right) \in E$,

$$
\beta_{l^{\prime}} \geq \max _{\substack{x_{1} \in G_{1, e} \\ V_{l}\left(x_{1}, x_{2}\right) \leq \beta_{l}}}\left(\max _{x_{1}^{\prime} \in R_{1, e}\left(x_{1}\right)} \min _{x_{2}^{\prime} \in R_{2, e}\left(x_{2}\right)} V_{l^{\prime}}\left(x_{1}^{\prime}, x_{2}^{\prime}\right)\right) .
$$

Let $\delta=\max \left(\sqrt{\beta_{1}}, \ldots, \sqrt{\beta_{|L|}}\right)$. Then, the relation $\mathcal{S}_{\delta} \subseteq Q_{1} \times Q_{2}$ defined by

$\mathcal{S}_{\delta}=\left\{\left(l_{1}, x_{1}, l_{2}, x_{2}\right) \mid l_{1}=l_{2}=l, V_{l}\left(x_{1}, x_{2}\right) \leq \beta_{l}\right\}$

is a $\delta$-approximate simulation relation of $T_{1}$ by $T_{2}$ and $T_{1} \preceq{ }_{\delta} T_{2}$.

Proof: Let $\left(l_{1}, x_{1}, l_{2}, x_{2}\right) \in \mathcal{S}_{\delta}$, then $l_{1}=l_{2}=l$ and $V_{l}\left(x_{1}, x_{2}\right) \leq \beta_{l}$. From equation (1), we have that $\left\|g_{l, 1}\left(x_{1}\right)-g_{l, 2}\left(x_{2}\right)\right\| \leq \sqrt{\beta_{l}} \leq \delta$. Hence, the first property of Definition 2.2 holds.

Let $\left(l_{1}, x_{1}\right) \stackrel{t}{\rightarrow}\left(l_{1}, x_{1}^{\prime}\right)$, then there exists an input $u_{1}($.$) and a function z_{1}($.$) such that z_{1}(0)=x_{1}$, $z_{1}(t)=x^{\prime}$ and for all $s \in[0, t], u_{1}(s) \in U_{1, l}$, $z_{1}(s) \in \operatorname{Inv}_{1, l}$ and

$$
\dot{z}_{1}(s)=f_{l, 1}\left(z_{1}(s), u_{1}(s)\right) .
$$

From Proposition 4.3, we know that there exists an input $u_{2}($.$) and a function z_{2}($.$) such that$ $z_{2}(0)=x_{2}$, and for all $s \in[0, t], u_{2}(s) \in U_{2, l}$,

$$
\dot{z}_{2}(s)=f_{l, 2}\left(z_{2}(s), u_{2}(s)\right)
$$

and $V\left(z_{1}(s), z_{2}(s)\right) \leq V\left(x_{1}, x_{2}\right) \leq \beta_{l}$. Then, assumption (a) of Theorem 4.4 insures that for all $s \in[0, t], z_{2}(s) \in \operatorname{Inv}_{l, 2}$. Let $x_{2}^{\prime}=z_{2}(t)$, we have $\left(l_{2}, x_{2}\right) \stackrel{t}{\rightarrow}\left(l_{2}, x_{2}^{\prime}\right)$ and since $V_{l}\left(x_{1}^{\prime}, x_{2}^{\prime}\right) \leq \beta_{l}$, $\left(l_{1}, x_{1}^{\prime}, l_{2}, x_{2}^{\prime}\right) \in \mathcal{S}_{\delta}$.

Let $\left(l_{1}, x_{1}\right) \stackrel{\tau}{\rightarrow}\left(l_{1}^{\prime}, x_{1}^{\prime}\right)$, then there exists $e=\left(l_{1}, l_{1}^{\prime}\right)$ such that $x_{1} \in G_{1, e}$ and $x_{1}^{\prime} \in R_{1, e}\left(x_{1}\right)$. Assumption (b) of Theorem 4.4 ensures that $x_{2} \in$ $G_{2, e}$. From assumption (d) of Theorem 4.4, we 
have that there exists $x_{2}^{\prime} \in R_{2, e}\left(x_{2}\right)$, such that $V_{l^{\prime}}\left(x_{1}^{\prime}, x_{2}^{\prime}\right) \leq \beta_{l^{\prime}}$ where $l^{\prime}=l_{1}^{\prime}$. Then, $\left(l_{2}, x_{2}\right) \stackrel{\tau}{\rightarrow}$ $\left(l_{2}^{\prime}, x_{2}^{\prime}\right)$ with $l_{2}^{\prime}=l^{\prime}$ and $\left(l_{1}^{\prime}, x_{1}^{\prime}, l_{2}^{\prime}, x_{2}^{\prime}\right) \in \mathcal{S}_{\delta}$. Therefore, $\mathcal{S}_{\delta}$ is a $\delta$-approximate simulation relation of $T_{1}$ by $T_{2}$.

Finally, let $\left(l_{1}, x_{1}\right) \in Q_{1}^{0}$, then $x_{1} \in I_{1, l}^{0}$ where $l=l_{1}$. From assumption (c) of Theorem 4.4, there exists $x_{2} \in I_{2, l}^{0}$, such that $V_{l}\left(x_{1}, x_{2}\right) \leq \beta_{l}$. Then, $\left(l_{2}, x_{2}\right) \in Q_{2}^{0}$ with $l_{2}=l$ and $\left(l_{1}, x_{1}, l_{2}, x_{2}\right) \in \mathcal{S}_{\delta}$. Then $T_{1} \preceq_{\delta} T_{2}$.

Assumption (d) can be interpreted as a condition of non-propagation of the approximation error through the reset maps. It is clear that the scalars $\beta_{1}, \ldots, \beta_{|L|}$ cannot be chosen independently. Thus, it is not necessarily the case that such numbers exist. There are two cases where we can guarantee easily the existence of these numbers. First, if we consider memoryless resets (i.e. $R_{1, e}\left(x_{1}\right)=R_{1, e}$ and $R_{2, e}\left(x_{2}\right)=R_{2, e}$ for all $e \in E$ ), then we can see that $\beta_{1}, \ldots, \beta_{l}$ can be chosen independently. Second, if the graph $(L, E)$ does not contain any cycle, then there is no circular dependency between $\beta_{1}, \ldots, \beta_{|L|}$ and thus it is easy to compute numbers such that the fourth assumption holds.

\subsection{Approximation of hybrid systems}

Based on Theorem 4.4, we can define a procedure to approximate a hybrid systems $H_{1}$ by another hybrid system $H_{2}$ with simpler continuous dynamics and to compute the precision of the approximate simulation relation of $T_{1}$ by $T_{2}$.

First, in each location $l \in L$, we approximate the continuous dynamics $F_{1, l}$ by a simpler continuous dynamics $F_{2, l}$. If $F_{1, l}$ is a large linear system, then $F_{2, l}$ may be chosen as a smaller linear system (Girard and Pappas, 2005a). If $F_{1, l}$ is a nonlinear system, then $F_{2, l}$ may be chosen as a linear system (Girard and Pappas, 2005b). The goal of this approximation is to reduce the complexity of analysis tasks such as reachability computation. Then, we compute a simulation function $V_{l}$ of the continuous dynamics $F_{1, l}$ by $F_{2, l}$. Note that such a function always exists if $F_{1, l}$ and $F_{2, l}$ are asymptotically stable. In the case of nonstable systems, a simulation function exists if the unstable subsystem of $F_{2, l}$ exactly simulates the unstable subsystem of $F_{1, l}$ (Girard and Pappas, 2005a).

The second part of the procedure consists in choosing the initial sets $I_{2, l}^{0}$ and the reset maps $R_{2, e}$ and computing scalars $\beta_{1}, \ldots, \beta_{|L|}$ satisfying the assumptions (c) and (d) of Theorem 4.4. Then, we set the invariants $\operatorname{Inv}_{2, l}=\mathcal{N}_{l}\left(\operatorname{Inv}_{1, l}, \beta_{l}\right)$ and the guards $G_{2, e}=\mathcal{N}_{l}\left(G_{1, e}, \beta_{l}\right)$ where $e=\left(l, l^{\prime}\right)$. From Theorem 4.4, we know that $T_{1} \preceq_{\delta} T_{2}$ with $\delta=\max \left(\sqrt{\beta_{1}}, \ldots, \sqrt{\beta_{|L|}}\right)$.

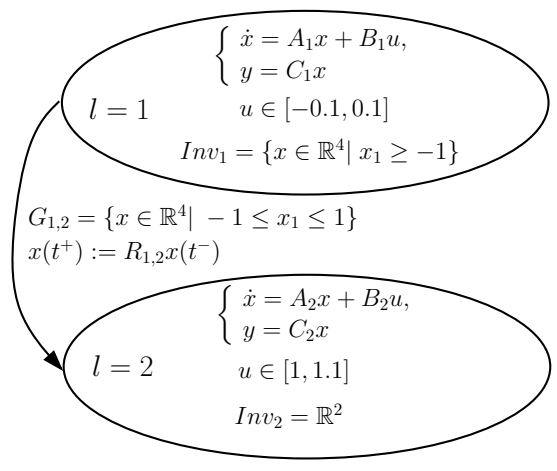

Fig. 1. Example of hybrid system

\section{EXAMPLE}

In this section, we illustrate our approximation framework in the context of a safety verification problem. Let us consider the hybrid system described in Figure 1. In each location, the continuous linear dynamics are given by the following matrices:

$$
\begin{gathered}
A_{1}=\left[\begin{array}{cccc}
-0.5 & 3 & 0 & 0 \\
-3 & -0.5 & 1 & 0 \\
0 & 0 & -0.7 & 8 \\
0 & 0 & -8 & -0.7
\end{array}\right], B_{1}=\left[\begin{array}{l}
0 \\
1 \\
0 \\
0
\end{array}\right], C_{1}^{T}=\left[\begin{array}{ll}
1 & 0 \\
0 & 1 \\
0 & 0 \\
0 & 0
\end{array}\right] \\
A_{2}=\left[\begin{array}{cc}
-0.5 & 1 \\
0 & -1
\end{array}\right], B_{2}=\left[\begin{array}{c}
-10 \\
5
\end{array}\right], C_{2}^{T}=\left[\begin{array}{ll}
1 & 0 \\
0 & 1
\end{array}\right] .
\end{gathered}
$$

The linear reset map is given by $R_{1,2}=\left[\begin{array}{llll}0 & 1 & 0 & 0 \\ 1 & 0 & 0 & 0\end{array}\right]$. The set of initial states is

$$
Q^{0}=\{1\} \times([4,5] \times[4,5] \times[0.9,1.1] \times\{0\}) .
$$

Let us consider the safety verification problem where the set of unsafe sets is

$\Pi_{U}=\{2\} \times\left\{y \in \mathbb{R}^{2} \mid\left(y_{1}+10\right)^{2}+\left(y_{2}+1\right)^{2} \leq 1\right\}$.

In order to solve the safety verification problem, we will use a two dimensional approximation of the continuous dynamics in location 1 , given by the following matrices:

$$
A_{1}^{\prime}=\left[\begin{array}{cc}
-0.5 & 3 \\
-3 & -0.5
\end{array}\right], B_{1}^{\prime}=\left[\begin{array}{l}
0 \\
1
\end{array}\right], C_{1}^{\prime}=\left[\begin{array}{ll}
1 & 0 \\
0 & 1
\end{array}\right] .
$$

The two dimensional dynamics in location 2 will be kept unchanged. The reset map of the approximate hybrid system is given by the matrix $R_{1,2}^{\prime}=\left[\begin{array}{ll}0 & 1 \\ 1 & 0\end{array}\right]$. The initial set is

$$
Q^{\prime 0}=\{1\} \times([4,5] \times[4,5]) .
$$

Simulation functions between the continuous dynamics are computed using the toolbox MATISSE. This essentially consists in solving a set of linear matrix inequalities and quadratic programs. Then, we compute $\beta_{1}$ and $\beta_{2}$ such that assumptions (c) and (d) of Theorem 4.4 hold. The invariant of location 1 and the guard of the transition $(1,2)$ are bloated according to these numbers. The precision of the approximate simulation relation between the original hybrid system and its approximation is $\delta=0.3877$. We computed the reachable sets of both systems using zonotope based reachability algorithms (Girard, 2005) implemented in MATISSE. We can see on Figure 2 

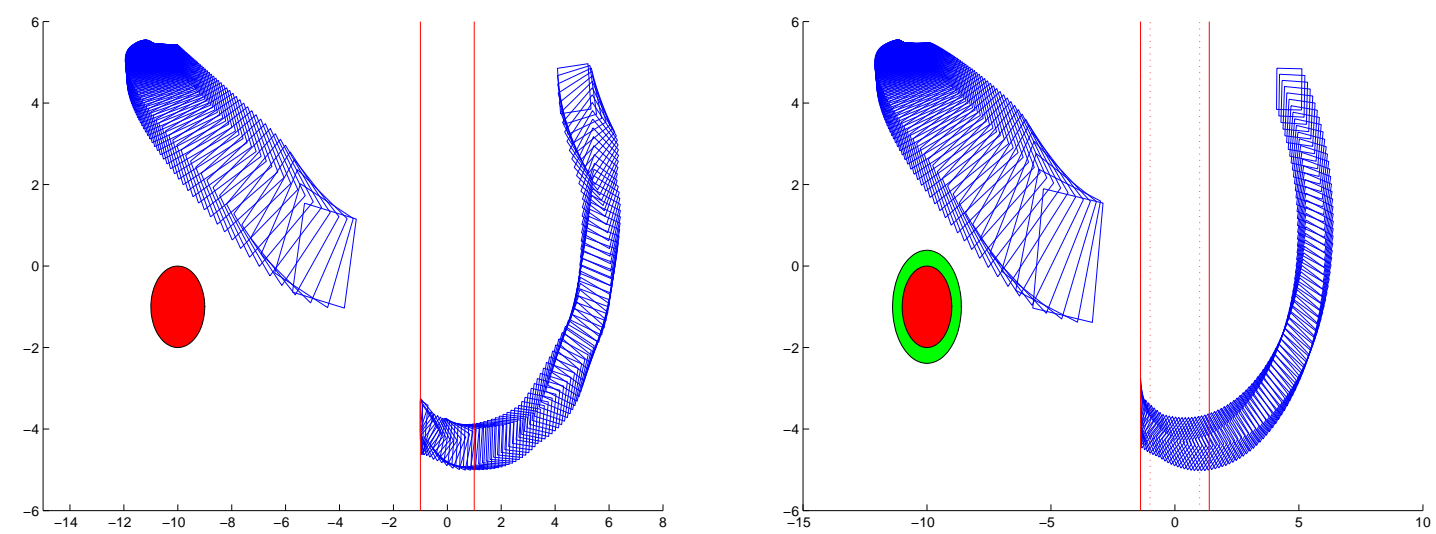

Fig. 2. Reachable sets of the original hybrid system (left) and of its approximation (right). We can see that the approximation allows to conclude that the original system is safe.

that the reachable set of the approximate hybrid system does not intersect the bloated unsafe set. Hence, this allows to conclude that the original hybrid system is safe.

\section{CONCLUSION}

In this paper, we extended the notion of approximate simulation relations to hybrid systems. We developed an effective characterization of approximate simulation relations based on simulation functions. We showed how our framework could be used to approximate hybrid systems and an example in the context of safety verification was shown. Future work includes developing more systematic methods to compute approximate simulation relations for hybrid systems as well as implementing these methods in MATISSE.

\section{REFERENCES}

Alur, R., T.A. Henzinger, G. Lafferriere and G.J. Pappas (2000). Discrete abstractions of hybrid systems. Proceedings of the IEEE 88, 971-984.

Alur, Rajeev, Costas Courcoubetis, Nicolas Halbwachs, Thomas A. Henzinger, Pei-Hsin Ho, Xavier Nicollin, Alfredo Olivero, Joseph Sifakis and Sergio Yovine (1995). The algorithmic analysis of hybrid systems.. Theor. Comput. Sci. 138(1), 3-34.

Clarke, E. M., O. Grumberg and D. A. Peled (2000). Model Checking. MIT Press.

de Alfaro, L., M. Faella and M. Stoelinga (2004). Linear and branching metrics for quantitative transition systems. In: ICALP'04. Vol. 3142 of LNCS. Springer. pp. 1150-1162.

Desharnais, J., V. Gupta, R. Jagadeesan and P. Panangaden (2004). Metrics for labelled markov processes. Theor. Comput. Sc. 318(3), 323-354.
Freeman, R. A. and P. V. Kokotovic (1996). Inverse optimality in robust stabilization. SIAM J. Control and Optimization 34(4), 13651391.

Girard, A. (2005). Reachability of uncertain linear systems using zonotopes. In: Hybrid Systems: Computation and Control. Vol. 3414 of LNCS. Springer. pp. 291-305.

Girard, A. and G. J. Pappas (2005a). Approximate bisimulations for constrained linear systems. In: Proc. IEEE Conference on Decision and Control and European Control Conference.

Girard, A. and G. J. Pappas (2005b). Approximate bisimulations for nonlinear dynamical systems. In: Proc. IEEE Conference on Decision and Control and European Control Conference.

Girard, A. and G. J. Pappas (2005c). Approximation metrics for discrete and continuous systems. Technical Report MS-CIS-05-10, Dept. of CIS, University of Pennsylvania.

Haghverdi, E., P. Tabuada and G. J. Pappas (2005). Bisimulation relations for dynamical, control, and hybrid systems. Theor. Comput. Sc. 342(2-3), 229-262.

Julius, A.A. (2006). Approximate abstraction of stochastic hybrid automata. In: Hybrid Systems: Computation and Control. Vol. 3927 of LNCS. Springer. pp. 318-332.

Julius, A.A., A. Girard and G.J. Pappas (2006). Approximate bisimulation for a class of stochastic hybrid systems. In: Proc. American Control Conference.

Liberzon, D., E. D. Sontag and Y. Wang (2002). Universal construction of feedback laws achieving ISS and integral-ISS disturbance attenuation. Systems and Control Letters 46, 111-127.

Pappas, G. J. (2003). Bisimilar linear systems. Automatica 39(12), 2035-2047. 